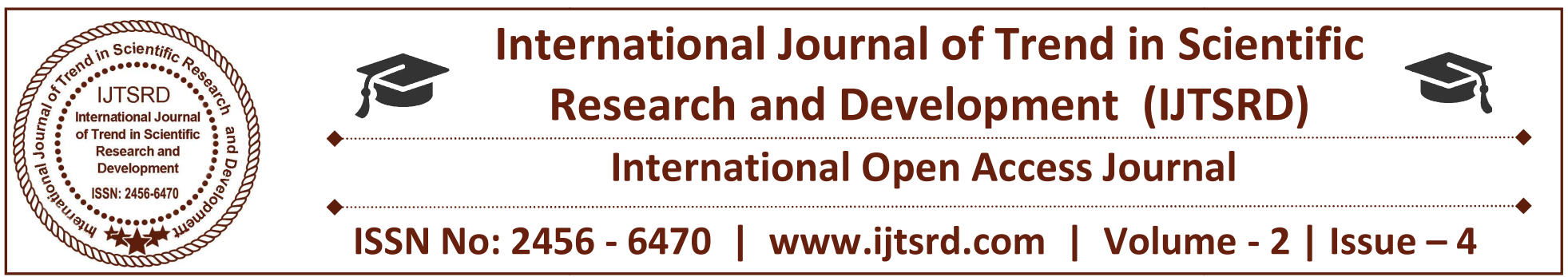

\title{
A Note On Applications of Cycles \& Trees
}

\author{
Chandrajeet Singh Yadav \\ Assistant Professor, Department of Mathematics \\ Vadodara Institute of Engineering, Vadodara, Gujarat, India
}

\begin{abstract}
Graph theory ia a branch of mathematics which deals with networks of points connected by lines called edges. The subject of graph theory had its beginnings in recreational math problems, but it has grown into a significant area of mathematical research with applications in chemistry, operations research, social sciences, and computer science. In this paper, we studied applications of path and circuits to the real world, so that author can understand the usefulness and importance of these things. Purpose of this paper is to provide an brief overview of applications area of cycles and trees.
\end{abstract}

vertices. Such graphs are known as traversable graphs.

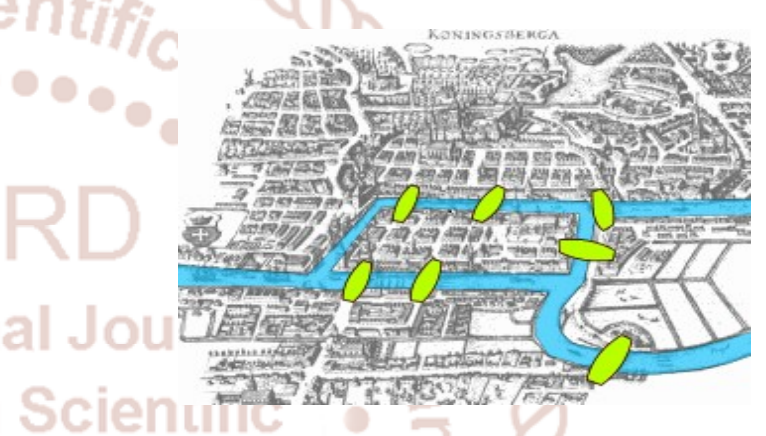

IMS Subject Classification : 05C30, 94C15

Keywords: Path, Eulerian circuit, Hamiltonian cycle, network topology

\section{INTRODUCTION}

Graph Theory's ${ }^{[2]}$ origin was the very famous Köingsberg seven bridges problem which was first found in 1735. Köingsberg was a city in Prussia situated on the Pregel River, which was served as the residence of the dukes' of Prussia in $16^{\text {th }}$ century. Today the city is named Kaliningrad.The Problem is "Does a walk n such a way that one can walk through all the seven bridges exactly once?" [5]

This problem was first solved by the prolific Swiss mathematician Leonhard Euler. Euler studied this problem and Construct a structure known as Euler graph. He proved that such walk was not possible through the seven bridges (each bridge cover exactly once). He observed that this problem can be solved when order of the vertex must be an even number. This must be true for all but at most two of the
Figure : 1.1

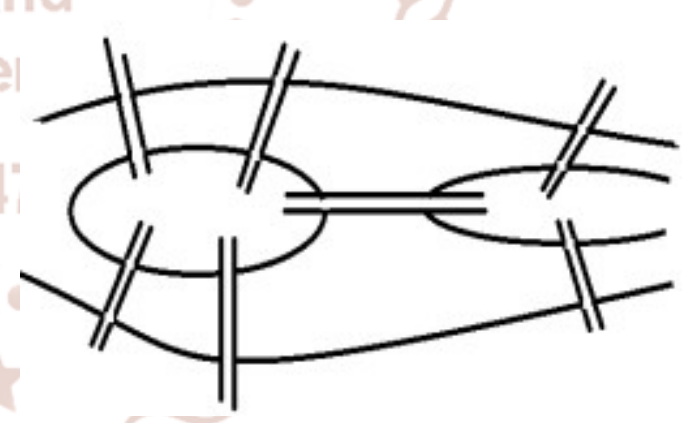

Figure : 1.2

It took 200 and odd years before the first book on graph theory was written, namely, "The orie der endlichen and unendlichenGraphen"(Ten buer, Leipzig 1736) by Köing in 1736. From 1736 till today, Graph theory becomes a popular and useful branch of mathematics, which has many applications in mathematics, computer science etc.

\section{Preliminaries}

Here we are showing some useful basic definitions which help for better understanding to the reader. 
2.1 Walk : In a pseudo graph an alternating sequences of vertices and edges, in which each edge is incident with the vertex immediately preceding it and the vertex immediately following it, is know an a Walk.

The number of edges in a walk is the length of the walk. A walk is closed if starting and final vertex is same; otherwise it is an open walk.

2.2 Connected graph : A graph is said to be connected if there exists a walk between any two of its vertices.

2.3 Path : A path is a walk in which all vertices are distinct.

2.4 Directed Path : A directed path or diapth ${ }^{[6]}$ is again a sequence of edges which connect a sequence of vertices keeping the fact that the edges all be directed in the same direction.

2.5 Trail : A trail is nothing but a walk in which every edge is different.

2.6 Circuit : A closed trail id known as a circuit.

2.7 Cycle : If in a circuit each vertex appears exactly once except starting and ending vertex then this is called a cycle. An n-cycle is a cycle with n vertices.

2.8 Eulerian Circuit : A circuit in a graph that contain every vertex and every edges of the given graph is known as Eulerian circuit and the graph is known as Euler graph. A graph is Eulerian if it is connected and each of its vertices having even degree. A graph which is having Eulerian trail but not having Eulerian circuit then it is known as semi Euler .

2.9 Connected graph : A graph is said to be connected if there exists a walk between any two of its vertices.

2.10 Hamiltonian Cycle : A cycle of a graph which contains all the vertices of the graph exactly once except starting and ending vertex then this cycle is known as Hamiltonian cycle and the graph is known as Hamiltonian graph.

2.11 Tree : A graph is a tree if it is connected and have no cycle.

2.12 Fat Tree ${ }^{[3]}$ : The fat tree network is a universal network for provably efficient communication. It was invented by Charles E. Leiserson of the Massachusetts Institute of Technology in 1985.

\section{Some Interesting Applications}

3.1 The Chinese Postman Problem ${ }^{[4]}$ : A postman, during his job, has to picks up mail at the post office then delivers it and finally returns to the post office. He must cover every single street at least once in the area given to him. He want to choose the way such that his walk is as small as possible. This is the famous Chinese postman problem. It is very similar to Köingsberg seven bridges problem. It was first considered by Mei-Ko Kwan in 1960, who was a Chinese mathematician. This Problem is also known as route inspection problem ${ }^{[8]}$. A version of the same problem was firstly solved by H.E. Dudeney in $1917^{[1]}$.

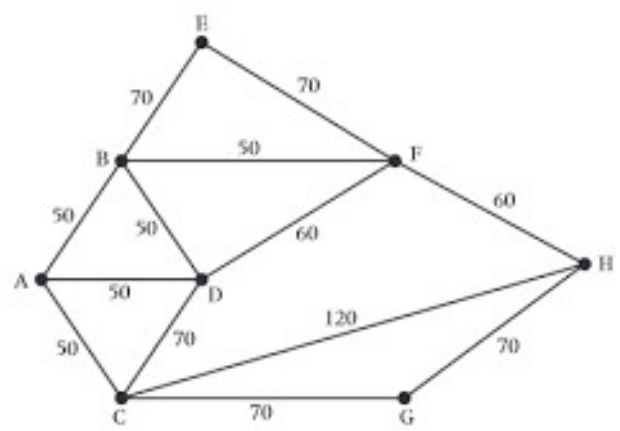

Figure : 3.1

Solution $^{[9]}$ of CPP is to find a Chinese postman route with least weight and we must walk along each edge at least once and in addition we must also walk along the least pairings of odd vertices on one extra occasion. To find such route first of all list all the odd vertices and then check the possible pair of odd vertices. Then for each pair find the edge with least weight. Then find the pairing so that the addition of weights is least. Then add these edges with least weight in the original graph. Then the sum of all edges added to the graph is the length of the Chinese postman route and finally a route corresponding to this minimum weight can then be easily found.

3.2 Network Topology : Let $\mathrm{G}(V, E)$ be a graph, in which each edge have a given capacity given by the function $\mathrm{C}: \mathrm{E} \rightarrow \mathrm{R}^{+}$, then this graph is known as network. Trees, cycles, grids etc. are the most popular network topologies which are useful in day to day practice. . Other suggested networks are just the combinations or derivatives of these. The path selection problem is much easier in trees which are a great benefit of tree since in every source-destination pair there is only one feasible simple path. However, since the root of a tree is usually a severe blockage, then fat trees have been used. These trees have the 
property that higher-level edges have a (much) larger capacity than lower-level edges. There are many network topologies are there. We are discussing some of them here.

3.2.1 Bus Topologies : If every computer and network devices connected with a single cable then this is known as bus topology. It is called linear bus topology if it has exactly two end points. Bus topology is cost effective, least cable required in it and used mostly in small networks. Drawback of this topology is if network traffic is heavy then its performance decreases and if cable has any kind of defect or failure then whole network fails.

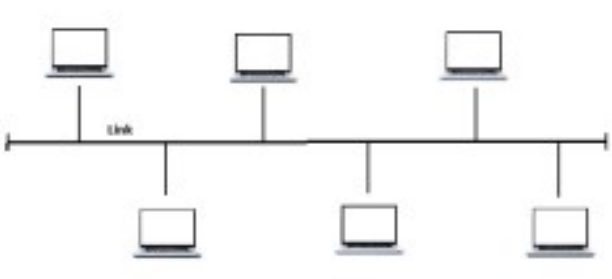

Figure : 3.2

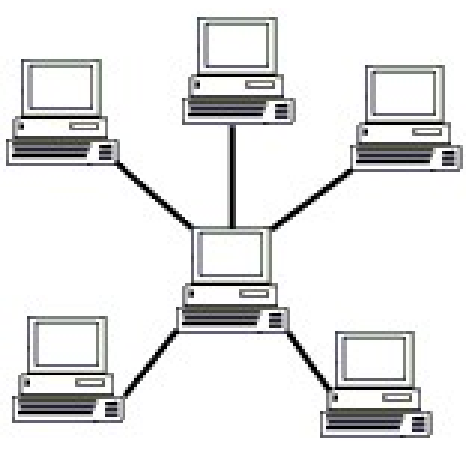

Figure : 3.4
3.3 Around The World Problem : Sir William Hamiltonian invented a game in 1857 namely the icosian game which is based on his works on quanterious. The object of this game is to get a Hamiltonian cycle along the edges of a dodecahedron (figure-4) in such a way that, except starting and ending vertices, every vertex is covered exactly once.

Around the world puzzle $e^{[7]}$ is one of the icosian game's variant. In this puzzle, the person is asked to fid a route along the dodecahedron that will pass through each angular point once, considering the fact that he can begin and end at anywhere. The similar puzzle is A Voyage round the world (figure 2). It considered of a wooden dodecahedron and a string. The 20 vertices with different cities in the world. Solution is shown in fugure-3 then whole network fails.
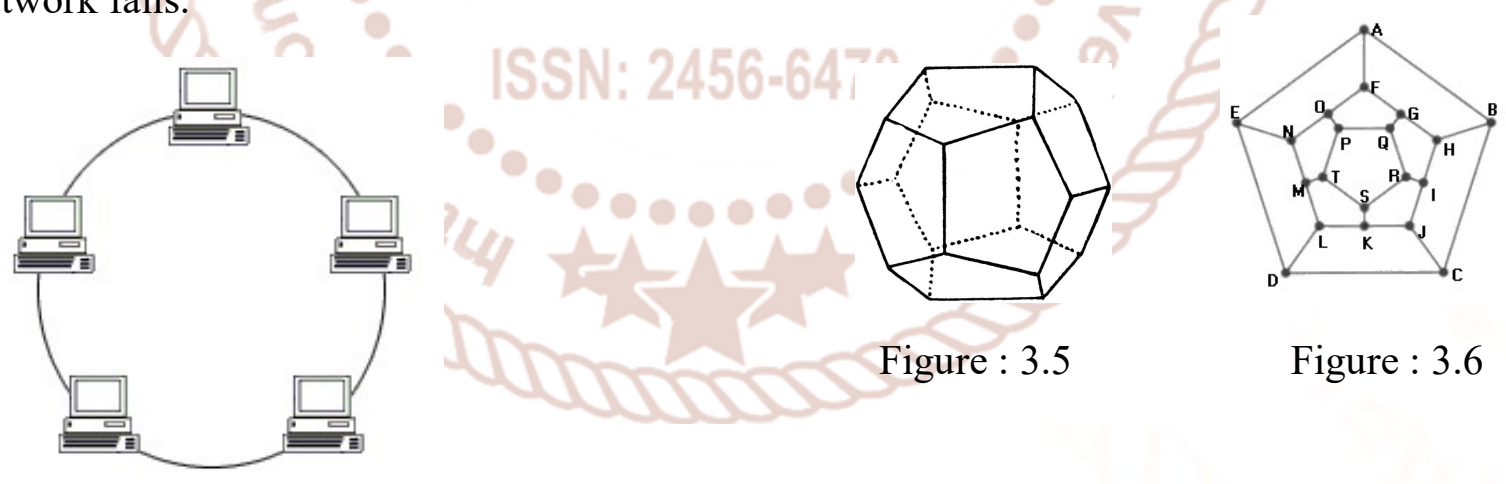

Figure : 3.5

Figure : 3.6

Figure : 3.3

3.2.3 Star Topology : If all the devices are connected to a single device, called hub, through cables then it is known as star topology. This hub is a central node. It is fast performer topology and easy in trouble shooting, set up and modification. The drawback is that its installation cost is very high and if hub is having any disturbance or problem the whole network effected.

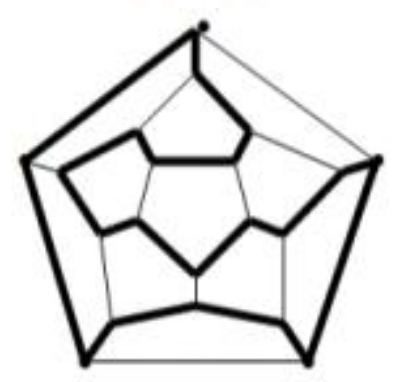

Figure : 3.7 


\section{Conclusion}

In this paper we discussed three different applications of graph theory. The first application which is Chinese postman problem is very useful in planning of bus routes, trash collection, and transmission line inspections etc. Network topologies are very important because it mediates in the outcome of changes in local communication. Network topologies are large element in network design theory. Around the world problem is useful and important application of graph theory which helps in scheduling the trip of a particular in which there are many cities and routes are included. The paper will help to understand that with the help graphs theory we can solve so many problems of our day to day life.

\section{References}

1) Angela Newing, " The life and Works of H.E. Dudeney”, Mathematical spectrum 21 (1988/89), $37-44$.

2) Bondy, J. A.; Murty, U. S. R. (1976). Graph Theory with Applications. North Holland. pp. 1221. ISBN 0-444-19451-7. Archived from the original on 2010-04-13.

3) Charles E. Leiserson, Fat Tree : universal networks for hardware-sfficient supercomputing, IEEE Transactions on Computers, Vol. 34, no. 10, Oct. 1985, pp. 892-901.

4) Edgar G. Goodaire, Michael M. Parmenter, Discrete Mathematics with Graph Theory, Prentice-Hall of India Pvt. Ltd., third edition, ISBN=978-81-203-3298-0

5) Euler, Leonhard (1736). "Solutio problematis ad geometriam situs pertinentis". Comment. Acad. Sci. U. Petrop 8, 128-40.

6) "Graph Structure Theory" Proceedings of AMSIMS-SIAM Joint Summer Research Conference on Grapg Theory, held on June 22 to July 05, 1991, P.-205.

7) Koshy Thomas, Discrete Mathematics with Applications, Academic press, An Imprint of Elsevier, ISBN-81-8147-887-8.

8) M.K.Kwan, " Graphic Programming using Odd and Even Points” Chinese Math 1 (1962) 273-277.

9) http://www.suffolkmaths.co.uk. 